\title{
COURBES SUR UN TRAIT ET MORPHISMES
}

\section{DE CONTRACTION}

\section{RAGNI PIENE}

\section{Introduction}

On étudie ici un certain type de schémas de dimension deux, qui sont de dimension relative 1 sur un trait (i.e. le spectre d'un anneau de valuation discrète) et qu'on appelle des courbes sur un trait.

Dans [7] S. Lichtenbaum a montré que des telles courbes regulières complètes sont projectives, et en a déduit une généralisation du critère de Castelnuovo pour l'existence des courbes exceptionelles sur une surface.

Le but de cet article est aussi de construire des morphismes de contraction, mais on ne suppose pas que les courbes sont regulières. On voit que des morphismes de contraction peuvent être construits à partir des diviseurs relatifs sur la courbe, et que, dans le cas où le trait est hensélien, tous les morphismes de contraction s'obtiennent de cette manière.

Ce sujet m'a été proposé par Michel Raynaud, je l'en remercie. Je remercie également Monsieur le rapporteur pour ses précieuses remarques qui m'ont aidée à améliorer ce travail.

\section{Morphismes d'un schéma dans un spectre homogène.}

On rappelle ici quelques résultats concernant le morphisme d'un schéma dans un spectre homogène, défini à partir d'un module inversible sur le schéma. (Pour un développement plus détaillé, voir EGA II [2].)

Soient $f: X \rightarrow Y$ un morphisme de schémas, $\mathscr{L}$ un $\mathcal{O}_{X}$-module inversible et $\mathscr{R}=\oplus_{n \geqq 0} \mathscr{R}_{n}$ une $\mathscr{O}_{Y}$-algèbre graduée quasi-cohérente. Soit $\mathscr{R}^{\prime}=\oplus_{n \geqq 0} \mathscr{L} \otimes n$, et supposons donné un morphisme de $\mathcal{O}_{\boldsymbol{Y}}$-algèbres graduées

$$
\psi: \mathscr{R} \rightarrow f_{*} \mathscr{R}^{\prime}=\oplus_{n \geqq 0} f_{*}(\mathscr{L} \otimes n) .
$$

Ces données définissent canoniquement un ouvert $G(\psi)$ de $X$ et un $Y$-morphisme

$$
r_{\mathscr{L}, \psi}: G(\psi) \rightarrow P
$$

où $P$ désigne le spectre homogène $\operatorname{Proj}(\mathscr{R})$.

Reçu Septembre 28, 1973. 
Si $\mathscr{R}=f_{*} \mathscr{R}^{\prime}$ et $\psi=\mathrm{id}$, on écrit $r_{\mathscr{L}}$ au lieu de $r_{\mathscr{L}, \text { id }}$.

Dans le cas où $Y$ est affine, $Y=\operatorname{Spec}(A)$, on a $\mathscr{R}=\tilde{R}, R$ étant une $A$-algèbre graduée. Alors $P=\operatorname{Proj}(R)$ a un recouvrement par les ouverts affines $P_{s}=\operatorname{Spec}\left(R_{(s)}\right)$ où $s$ est un élément homogène de $R_{+}$et $R_{(s)}$ est l'anneau des éléments de degré 0 de $R_{s}$. De plus,

$$
r_{\mathscr{L}, \psi}^{-1}\left(P_{s}\right)=X_{\psi(s)}=\{x \in X ; \psi(s)(x) \neq 0\},
$$

et $r_{\mathscr{L}, \varphi} \mid X_{\psi(g)}$ correspond à l'homomorphisme d'algèbres

$$
\Gamma\left(P_{s}, \mathcal{O}_{P}\right)=R_{(s)} \rightarrow \Gamma\left(X_{\psi(s)}, \mathcal{O}_{X}\right)
$$

qui envoie un élément $x / s^{n}$ dans $\left(x \mid X_{\psi(s)}\right)\left(\psi(s) \mid X_{\psi(s)}\right)^{-n}$.

Dans le cas où les ouverts $X_{\psi(s)}, s$ homogène dans $R_{+}$, forment un recouvrement de $X$, on a $G(\psi)=X$, et on dit alors que $r_{\mathscr{L}, \psi}$ est partout défini. Autrement dit, $r_{\mathscr{L}, \varphi}$ est partout défini si et seulement si, pour tout $x$ dans $X$, il existe $n>0$ et $s \in R_{n}$ tel que $\psi(s)(x) \neq 0$.

Supposons que $X$ soit quasi-compact et quasi-séparé, et que $\mathscr{L}$ soit tel que $r_{\mathscr{L}}: X \rightarrow P=\operatorname{Proj}(R)$ soit partout défini. Les homomorphismes d'algèbres qui définissent localement $r_{\mathscr{L}}$ sont alors des isomorphismes (EGA, I [1, 6.8.2]), de sorte que l'homomorphisme canonique

$$
\mathcal{O}_{P} \rightarrow\left(r_{\mathscr{L}}\right)_{*} \mathcal{O}_{X}
$$

est un isomorphisme. Donc $r_{\mathscr{L}}$ est schématiquement dominant, ce qui entraine que $P$ est intègre (i.e. réduit et irréductible) si $X$ l'est.

Plus généralement, pour tout $s$ homogène dans $R_{+}$, on a des isomorphismes canoniques (EGA, I $[1,6.8 .2]$ )

$$
\Gamma\left(P_{s},\left(r_{\mathscr{L}}\right)_{*}(\mathscr{L} \otimes m)\right)=\Gamma\left(X_{s}, \mathscr{L} \otimes m\right) \rightarrow R(m)_{(s)} \quad \text { pour tout } m \in \mathrm{Z},
$$

et par définition des $\mathcal{O}_{P}$-modules $\mathcal{O}_{P}(m)$, on a

$$
\Gamma\left(P_{s}, \mathcal{O}_{P}(m)\right)=R(m)_{(s)},
$$

de sorte qu'on obtient des isomorphismes

$$
\left(r_{\mathscr{L}}\right)_{*}(\mathscr{L} \otimes m) \rightarrow \mathcal{O}_{P}(m) \quad \text { pour tout } m \in \mathbf{Z} .
$$

Si $X$ est quasi-compact et $r_{\mathscr{L}}$ partout défini, il existe un nombre fini d'eléments homogènes $s \in R_{+}$tel que $X=\cup_{s} X_{s}$. On peut en outre supposer que tous les $s$ ont le même degré $d>0$. Les $\mathcal{O}_{P}$-modules $\mathcal{O}_{P}(m d)$ sont alors inversibles, d'inverses $\mathcal{O}_{P}(-m d)$, sur l'ouvert $\cup_{s \in R_{d}} P_{s}$, pour tout $m \in Z$ (EGA, II [2,2.5.17]). Il en résulte qu'on a des isomorphismes canoniques $[6, I, 2.1]$

$$
\left(r_{\mathscr{L}}\right)^{* \mathcal{O}_{P}}(m d) \rightarrow \mathscr{L} \otimes m d, \quad m \in \mathbf{Z} .
$$


Le spectre homogène d'une $A$-algèbre graduée de type fini est un $A$ schéma de type fini - l'implication dans l'autre sens n'étant pas vraie en général. Dans le cas où $P=\operatorname{Proj}(R)$ est construit à partir d'un module inversible $\mathscr{L}$ sur un $A$-schéma $X$, on va montrer, sous des conditions sur $X$ et $\mathscr{L}$, que $P$ est projectif et aussi qu'on peut en déduire que $R$ est une $A$-algèbre de type fini. Ce résultat est étroitement lié à ce qu'a fait Zariski [11, Theorem 4.2] pour les variétés algébriques.

Proposition 1. Soient $Y=\operatorname{Spec}(A)$ un schéma noethérien affine, $f: X \rightarrow Y$ un morphisme propre de schémas et $\mathscr{L}$ un $\mathcal{O}_{X}$-module inversible tel que

$$
r_{\mathscr{L}}: X \rightarrow P=\operatorname{Proj}\left(\oplus_{n \geqq 0} \Gamma(X, \mathscr{L} \otimes n)\right)
$$

soit partout défini. Alors $P$ est un $Y$-schéma projectif et $R=\oplus_{n \geqq 0} \Gamma(X, \mathscr{L} \otimes n)$ est une A-algèbre de type fini.

DÉmonstration. $r_{\mathscr{L}}$ est dominant et propre (car $f$ est propre et $P$ est séparé), donc $r_{\mathscr{L}}$ est surjectif. Il s'en suit que le morphisme $P \rightarrow Y$ est universellement fermé (EGA, II [2,5.4.9]).

$P=r_{\mathscr{L}}(X)$ est quasi-compact. Soient $\left\{s_{i}\right\}_{1}^{m}$ des éléments homogènes de l'anneau gradué $R=\oplus_{n \geqq 0} \Gamma(X, \mathscr{L} \otimes n)$ tels que $P=\bigcup_{i=1}^{m} P_{s_{i}}$. On peut supposer que tous les $s_{i}$ ont le même degré $d>0$. Soient $R^{\prime}$ la sous-algèbre graduée de

$$
R^{(d)}=\oplus_{n \geqq 0} R_{n d}
$$

engendrée sur $A$ par $\left\{s_{i}\right\}$ et $\psi: R^{\prime} \rightarrow R^{(d)}$ l'inclusion. La composition de $\operatorname{Proj}(\psi)$ avec l'isomorphisme $P \cong \operatorname{Proj}\left(R^{(d)}\right)$ définit un morphisme affine

$$
\Phi: P \rightarrow P^{\prime}=\operatorname{Proj}\left(R^{\prime}\right)
$$

tel que $\Phi \circ r_{\mathscr{L}}=r_{\mathscr{L} \otimes d, \psi}$. Soit $r^{\prime}=r_{\mathscr{L} \otimes d, \psi} . P^{\prime}$ est un schéma noethérien étant de type fini sur un schéma noethérien - et $r^{\prime}$ est propre, donc

$$
r^{\prime} \mathcal{O}_{X} \cong \Phi_{*}\left(r_{\mathscr{L}}\right)_{*} \mathcal{O}_{X} \cong \Phi_{*} \mathcal{O}_{P}
$$

est une $\mathcal{O}_{P}$-algèbre finie. Comme $\Phi$ est affine, cela veut dire que $P$ est un $P^{\prime}$-schéma fini. Donc $P$ est de type fini sur $Y$.

Le $\mathcal{O}_{P}$-module $\mathcal{O}_{P}(d)$ est inversible $\left(\mathrm{EGA}\right.$, II [2,2.5.17]) et $\mathcal{O}_{P}(d) \cong$ $\left(r_{\mathscr{L}}\right)_{*}(\mathscr{L} \otimes d)$, donc $\mathcal{O}_{P}(d)$ est ample sur $P$ (EGA, II $\left.[2,4.5 .2]\right) . P$ est alors un $Y$-schéma quasi-projectif et universellement fermé, donc projectif.

Pour montrer la dernière assertion, rappellons le résultat suivant (EGA, III [3,2.3.4(ii)]): soient $q: P \rightarrow Y$ un morphisme projectif, $\mathscr{S}=$ $\oplus_{n \in Z} \mathscr{S}_{n}$ une $\mathscr{O}_{P}$-algèbre graduée telle que les $\mathscr{S}_{n}$ sont des $\mathcal{O}_{P}$-modules 
cohérents et telle qu'il existe $d>0$ avec $\mathscr{S}_{d}$ inversible et ample, d'inverse $\mathscr{S}_{-d}$. Alors $\mathscr{R}=\oplus_{n \geqq 0} q_{*} \mathscr{S}_{n}$ est une $\mathcal{O}_{Y}$-algèbre graduée de type fini.

On a vu que $\mathscr{S}=\oplus_{n \in Z} \mathcal{O}_{P}(n)$ satisfait à ces conditions, donc

$$
\mathscr{R}=\oplus_{n \geqq 0} q_{*} \mathcal{O}_{P}(n)=\oplus_{n \geqq 0} f_{*}(\mathscr{L} \otimes n)
$$

est une $\mathcal{O}_{Y}$-algèbre de type fini - i.e. $R$ est une $A$-algèbre de type fini.

\section{Diviseurs relatifs.}

On rappelle qu'un diviseur sur un schéma $X$ est défini par un idéal inversible de l'anneau total des fractions du faisceau structural $\mathcal{O}_{X}$. Soit $\operatorname{Div}(X)$ le groupe des diviseurs sur $X$. Si $D \in \operatorname{Div}(X)$, on pose $\mathcal{O}_{X}(D)=$ $\mathscr{I}_{X}(D)^{-1}$, où $\mathscr{I}_{X}(D)$ est l'idéal qui définit $D$. On dit que $D$ est effectif, noté $D \geqq 0$, si $\mathscr{I}_{X}(D)$ est un idéal de $\mathcal{O}_{X}$. L'ensemble des diviseurs effectifs est noté $\operatorname{Div}^{+}(X)$.

Si $D \in \operatorname{Div}^{+}(X), D$ définit un sous-schéma fermé régulièrement immergé dans $X$ et de codimension 1 . Inversement, un tel sous-schéma définit un diviseur effectif et un seul. On peut donc identifier un diviseur effectif avec le sous-schéma fermé qu'il définit (EGA, IV [4,21.2.12]).

Soit $f: X \rightarrow Y$ un morphisme plat et localement de présentation finie, et soit $D \in \operatorname{Div}^{+}(X)$. Si le sous-schéma défini par $D$ est plat sur $Y$, on dit que $D$ est un diviseur (effectif) relatif à $Y$. On note $\operatorname{Div}^{+}(X / Y)$ l'ensemble de ces diviseurs.

Pour tout morphisme $Y^{\prime} \rightarrow Y$, on obtient une application canonique

$$
\operatorname{Div}^{+}(X / Y) \rightarrow \operatorname{Div}^{+}\left(X \times_{Y} Y^{\prime} / Y^{\prime}\right) .
$$

En particulier, tout diviseur relatif induit un diviseur effectif sur chaque fibre de $X$ sur $Y$.

Proposition 2. Soient $A$ un anneau local noethérien, $Y=\operatorname{Spec}(A)$, $f: X \rightarrow Y$ un morphisme plat et propre. On suppose en outre que toutes les composantes irréductibles des fibres de $f$ soient de dimension 1 (i.e. que les fibres de $f$ soient des courbes algébriques). Alors pour tout $D \in \operatorname{Div}^{+}(X / Y)$, $r_{\mathcal{O}_{X}(D)}$ est partout défini.

Démonstration. On remarque d'abord que, si $D=0$, il n'y a rien à démontrer. (En fait, pour tout diviseur $D$ tel que $\mathcal{O}_{X}(D) \cong \mathcal{O}_{X}$,

$$
\operatorname{Proj}\left(\oplus_{n \geqq 0} \Gamma\left(X, \mathcal{O}_{X}(D) \otimes n\right)\right)=\operatorname{Spec}\left(f_{*} \mathcal{O}_{X}\right),
$$

et $r_{O_{X}(D)}$ n'est autre qu'une factorisation de Stein de $f$.) 
Soit $D \in \operatorname{Div}^{+}(X / Y)$, et considérons la suite exacte

$$
0 \rightarrow \mathscr{I}_{X}(D) \rightarrow \mathcal{O}_{X} \rightarrow \mathcal{O}_{D} \rightarrow 0 .
$$

$D$ induit un diviseur effectif sur chaque fibre de $f$, qui sont des courbes algébriques. $D$ est donc quasi-fini sur $Y$ et propre, donc on a $D=\operatorname{Spec}(B)$, ou $B$ est une $A$-algèbre finie. Il en résulte que tout $\mathcal{O}_{D}$-module inversible est trivial et que $H^{1}\left(D, \mathcal{O}_{D}\right)=0$.

Soit $\mathscr{L}=\mathcal{O}_{X}(D)$. Par tensorisation on obtient de $\left({ }^{*}\right)$ des suites exactes

$$
0 \rightarrow \mathscr{L} \otimes(n-1) \rightarrow \mathscr{L}^{\otimes n} \rightarrow \mathcal{O}_{D} \rightarrow 0 .
$$

En particulier, l'injection $\mathcal{O}_{X} \rightarrow \mathscr{L}$ de $\left({ }^{*}\right)_{1}$, correspond à une section $s_{1}$ de $\mathscr{L}$ au-dessus de $X$ telle que $s_{1}(x) \neq 0$ si $x \notin D$.

Montrons qu'il existe $d>0$ et $s_{2} \in \Gamma(X, \mathscr{L} \otimes d)$ tel que $s_{2}(x) \neq 0$ pour tout $x \in D$, ce qui achèvera la démonstration de la proposition.

Pour tout $n,\left({ }^{*}\right)_{n}$ donne une suite exacte

$$
\begin{aligned}
0 \rightarrow & \Gamma(X, \mathscr{L} \otimes n-1) \rightarrow \Gamma(X, \mathscr{L} \otimes n) \rightarrow \Gamma\left(D, \mathcal{O}_{D}\right) \rightarrow \\
& \stackrel{\delta_{n}}{\rightarrow} H^{\mathbf{1}}\left(X, \mathscr{L}^{\otimes n-1}\right) \rightarrow H^{1}(X, \mathscr{L} \otimes n) \rightarrow 0 .
\end{aligned}
$$

Le lemme suivant montre que, pour $n$ assez grand, $\delta_{n}$ est le morphisme 0 .

Lemme 1. Soient $A$ un anneau, et $\left\{E_{i}\right\}_{i \geq 0}$ des A-modules noethériens. Supposons donnés des morphismes surjectifs $E_{i} \rightarrow E_{i+1}$ pour tout $i$. Alors ce sont des isomorphismes pour $i \gg 0$.

Démonstration. Soient $N_{i}=\operatorname{Ker}\left(E_{i} \rightarrow E_{i+1}\right)$ et $\alpha_{i}: E_{0} \rightarrow E_{i}$ la surjection composée. $\left\{\alpha_{i}{ }^{-1}\left(N_{i}\right)\right\}_{i \geqq 1}$ est une suite croissante de sous-modules de $E_{0}$, donc stationnaire pour $i$ grand. Comme

$$
\operatorname{Ker}\left(\alpha_{i+1}\right)=\alpha_{i}^{-1}\left(N_{i}\right),
$$

on a $\operatorname{Ker}\left(\alpha_{i+1}\right)=\operatorname{Ker}\left(\alpha_{i}\right)$ pour $i$ grand, et le lemme en résulte.

Soit donc $d>0$ tel que $\Gamma(X, \mathscr{L} \otimes d) \rightarrow \Gamma\left(D, \mathcal{O}_{D}\right)$ soit surjectif. On peut choisir $s_{2} \in \Gamma(X, \mathscr{L} \otimes d)$ telle que $s_{2}$ ait pour image dans $\Gamma\left(D, \mathcal{O}_{D}\right)$ l'élément unité de cet anneau. Alors $s_{2}(x) \neq 0$ pour $x \in D$, et on a montré que $r_{\mathscr{L}}$ est partout défini.

De cette propostion on déduit le critère d'amplitude bien connu des diviseurs sur une courbe algébrique, de façon analogue à ce qu'on fait pour démontrer le critère d'amplitude de Nakai (voir p. ex. [6]) et un critère d'amplitude des diviseurs sur un schéma sur un anneau local. 
CoRollaIRE 1. Soient $X$ une courbe algébrique complète sur un corps $k$ et $D$ un diviseur effectif sur $X$. $D$ est ample si et seulement si $D$ rencontre chacune des composantes irréductibles de $X$.

Démonstration. Soit $r=r_{\mathcal{O}_{X}(D)}$. Par la proposition 2, $r$ est partout défini.

Soient $\left\{X_{i}\right\}$ les composantes irréductibles de $X$. On va d'abord montrer que si $D \cap X_{i} \neq \varnothing$ pour tout $i$, alors $D$ est ample, ou - ce qui revient au même - que $r$ est un isomorphisme. Comme $r$ est propre et $r_{*} \mathcal{O}_{X} \cong \mathcal{O}_{P}$, il suffit de montrer que $r$ est quasi-fini. Montrons donc que, pour tout $i$, $r\left(X_{i}\right)$ ne peut pas être un seul point.

Choisissons $x \in D \cap X_{i}$ et $y \in X_{i}, y \notin D$. La démonstration de la proposition 2 montre qu'il existe une section $s_{1}$ de $\mathcal{O}_{X}(D)$ telle que $s_{1}(x)=0$, $s_{1}(y) \neq 0$. Donc $r(x) \neq r(y)$.

Inversement, s'il existe $X_{i}$ telle que $D \cap X_{i}=\varnothing$, montrons que l'image de $X_{i}$ par $r$ est un point, donc que $r$ ne peut pas être un isomorphisme.

Soient $s$ une section de $\mathcal{O}_{X}(D), s_{i}$ son image dans

$$
\Gamma\left(X_{i}, \mathcal{O}_{X}(D) \mid X_{i}\right)=\Gamma\left(X_{i}, \mathcal{O}_{X_{i}}\right) \text {. }
$$

Comme $\Gamma\left(X_{i}, \mathcal{O}_{X_{i}}\right)$ est un anneau artinien, si $s_{i}$ s'annule en un point $x$ de $X_{i}$, elle s'annule en tout point de $X_{i}$, ce qui montre que tous les points de $X_{i}$ ont la même image par $r$.

CorollaIRE 2. Soient $A$ un anneau local noethérien, $f: X \rightarrow Y=$ $\operatorname{Spec}(A)$ un morphisme plat et propre tel que les fibres de $f$ soient des courbes algébriques, $D \in \operatorname{Div}^{+}(X / Y)$. Alors $D$ est ample si et seulement si $D$ rencontre chacune des composantes irréductibles de la fibre fermée de $f$.

Démonstration. En fait, $D$ est ample si et seulement si la restriction de $D$ à la fibre fermée est ample (EGA, III [3, 4.7.1]).

\section{Morphismes de contraction entre des courbes sur un trait.}

Définition 1. Soit $S$ un trait (c'est-à-dire le spectre d'un anneau de valuation discrète). Une courbe sur $S$ est un schéma séparé $X$ et un morphisme $f: X \rightarrow S$ plat, de type fini et tel que les fibres de $f$ soient des courbes algébriques. On dit que la courbe est complète si $f$ est propre.

Définition 2. Soit $g: X \rightarrow X^{\prime}$ un morphisme de courbes complètes sur un trait $S$. Soient $\left\{C_{i} ; i \in I\right\}$ les composantes irréductibles de la fibre fermée de $X$ au-dessus de $S$ et $J$ un sous-ensemble de $I$. 
On appelle $g$ une contraction de $\left\{C_{j} ; j \in J\right\}$ si les conditions suivantes sont vérifiées:

(i) $g \mid U$ est un isomorphisme de $U$ sur $P-g\left(\bigcup_{j \in J} C_{j}\right)$, où on a posé $U=X-\cup_{j \in J} C_{j}$.

(ii) $g\left(C_{j}\right)$ est un point (fermé) de $X^{\prime}$, pour tout $j \in J$.

(iii) $\mathcal{O}_{X^{\prime}} \cong g_{*} \mathcal{O}_{X}$.

On dit que $g$ est une contraction projective si $X^{\prime}$ est projectif.

Remarque 1. Le nombre des points de $g\left(\cup_{j \in J} C_{j}\right)$ est égal au nombre des composantes connexes de $\bigcup_{j \in J} C_{j}$. (C'est une conséquence du "théorème de connexion 》 de Zariski (EGA, III $[3,4.3 .2])$.)

Proposition 3. Soit $g: X \rightarrow X^{\prime}$ un morphisme de courbes intègres et complètes sur un trait $S$. Supposons que g soit une contraction. Si $X$ est normal, $X^{\prime}$ l'est aussi.

Démonstration. En fait, le résultat est vrai si on suppose seulement que $g$ soit un morphisme propre et birationnel de schémas noethériens intègres tel que $\mathcal{O}_{X^{\prime}} \cong g_{*} \mathcal{O}_{X}$.

Soient $\mathscr{R}(X)$ (resp. $\mathscr{R}\left(X^{\prime}\right)$ ) le faisceau des fonctions rationnelles sur $X$ (resp. $X^{\prime}$ ) et $q: Y \rightarrow X^{\prime}$ le normalisé de $X^{\prime}$ par rapport à $\mathscr{R}\left(X^{\prime}\right)$. Comme $X$ est normal, $g$ se factorise - soit $h: X \rightarrow Y$ le morphisme tel que $q \circ h=g$. Mais alors $\mathcal{O}_{Y} \cong h_{*} \mathcal{O}_{X}$ (EGA, III [3, 4.3.12]), donc $q_{*} \mathcal{O}_{Y} \cong$ $g_{*} \mathcal{O}_{X} \cong \mathcal{O}_{X^{\prime}}$. Comme $q$ est un morphisme affine, cela entraîne que $q$ est un isomorphisme.

Proposition 4. Soit $X$ une courbe complète sur un trait $S$. Supposons que $g: X \rightarrow X^{\prime}$ et $h: X \rightarrow X^{\prime \prime}$ soient des contractions d'une famille $\left\{C_{j} ; j \in J\right\}$. Il existe alors un isomorphisme unique $\varphi: X^{\prime} \rightarrow X^{\prime \prime}$ tel que $\varphi \circ g=h$.

Démonstration. On sait qu'il existe $\varphi: X^{\prime} \rightarrow X^{\prime \prime}$ et $\psi: X^{\prime \prime} \rightarrow X^{\prime}$ tels que $\varphi \circ g=h$ et $\psi \circ h=g$ (EGA, II $[2,8.11 .1])$. Comme $g$ et $h$ sont des épimorphismes dans la catégorie des $S$-schémas (EGA, I $[1,5.4 .6]$ ), on obtient $\psi \circ \varphi=\mathrm{id}_{X^{\prime}}$ et $\varphi \circ \psi=\mathrm{id}_{X^{\prime \prime}}$. L'unicité de $\varphi$ résulte aussi du fait que $g$ est un épimorphisme.

On va maintenant donner deux théorèmes qui caractérisent les contractions projectives d'une courbe complète. 
ThÉoRÈme 1. Soient $f: X \rightarrow S$ une courbe complète sur un trait $S$, $\left\{C_{i} ; i \in I\right\}$ les composantes irréductibles de la fibre fermée de $f, D \in$ $\operatorname{Div}^{+}(X / S)$. Supposons que la restriction de $D$ à la fibre générique de $f$ soit ample. Alors

$$
r_{\mathcal{O}_{X}(D)}: X \rightarrow P=\operatorname{Proj}\left(\oplus_{n \geq 0} \Gamma\left(X, \mathcal{O}_{X}(D) \otimes n\right)\right)
$$

est une contraction projective de la famille $\left\{C_{j} ; j \in I\right.$ et $\left.C_{j} \cap D=\varnothing\right\}$.

THÉORÈme 2. Soit $g: X \rightarrow X^{\prime}$ un morphisme de courbes complètes sur un trait $S$. On suppose que $g$ soit une contraction projective de la famille $\left\{C_{j} ; j \in J\right\}$. Il existe alors $D \in \operatorname{Div}^{+}(X / S)$ et un isomorphisme

$$
\varphi: X^{\prime} \rightarrow P=\operatorname{Proj}\left(\oplus_{n \geqq 0} \Gamma\left(X, \mathcal{O}_{X}(D) \otimes n\right)\right)
$$

tels que $r_{\mathcal{O}_{X}(D)}=\varphi \circ g$.

Démonstration DU Théorème 1. Soient $s$ le point fermé de $S, t$ son point générique,

$$
R=\oplus_{n \geqq 0} \Gamma\left(X, \mathcal{O}_{X}(D) \otimes n\right),
$$

$r=r_{\mathcal{O}_{X}(D)}$. Les propositions 1 et 2 montrent que $r$ est un morphisme propre et surjectif de la courbe $X$ dans le $S$-schéma projectif $P=\operatorname{Proj}(R)$. Il faut montrer que $P$ est une courbe sur $S$ et que $r$ est une contraction, i.e. que les conditions (i), (ii) et (iii) de la définition 2 sont vérifiées.

D'après la construction du morphisme $r$, (iii) est vérifiée. Montrons que (i) et (ii) le sont aussi et qu'on en déduit que $P$ est une courbe sur $S$.

Par hypothèse, $D_{t}$ est un diviseur ample sur $X_{t}$. La construction du morphisme $r_{\mathcal{O}_{X}(D)}$ commute aux changements de base plats, donc $r \mid X_{t}$ : $X_{t} \rightarrow P_{t}$ est un isomorphisme.

Comme dans la démonstration du corollaire 1 , on voit que $r\left(C_{j}\right)$ est un point de $P$ si $j \in J$, où

$$
J=\left\{i \in I ; D \cap C_{i}=\varnothing\right\},
$$

et que $r\left(C_{i}\right)$ est une courbe dans $P$ si $i \in I-J$.

Soit $U=X-\cup_{j \in J} C_{j}$. De ce qui précède on déduit que $r \mid U: U \rightarrow P$ est un morphisme quasi-fini. Puisque $P$ est noetherien, que $r$ est propre et $\mathcal{O}_{P} \cong r_{*} \mathcal{O}_{X}$, le "théorème de connexion 》 de Zariski (EGA, III [3, 4.3.2]) donne que les fibres de $r$ sont connexes. Done $r^{-1}(r(U))=U$, i.e. le diagramme suivant est cartésien:

$$
\begin{aligned}
& X \rightarrow P \\
& \vartheta \\
& U \rightarrow r(U)
\end{aligned}
$$


Cela montre que $r \mid U: U \rightarrow r(U)$ est propre, donc fini et en particulier affine. Mais alors c'est un isomorphisme, d'après la construction de $r$.

Pour montrer que $P$ est plat sur $S$, il suffit de voir que l'anneau de l'ouvert affine $P_{a}$ est sans torsion sur l'anneau de valuation discrète $\Gamma\left(S, \mathcal{O}_{S}\right)$, pour tout $a \in R_{+}$homogène. Mais $\Gamma\left(P_{a}, \mathcal{O}_{P}\right)=\Gamma\left(X_{a}, \mathcal{O}_{X}\right)$, et on sait que $\Gamma\left(X_{a}, \mathcal{O}_{X}\right)$ est sans torsion sur $\Gamma\left(S, \mathcal{O}_{S}\right)$ car $X$ est plat sur $S$.

Le fait que $P$ soit plat sur $S$ donne que toutes les composantes irréductibles de $P_{s}$ sont de dimension 1 , donc $P$ est une courbe sur $S$.

REMARque 2. Si $X$ est une courbe complète intègre sur un trait $S$ et $D$ un élément de $\operatorname{Div}^{+}(X / S)$, alors $D_{t}$ est ample si et seulement si $D \neq 0$, ou encore, si et seulement si $\mathcal{O}_{X}(D)$ n'est pas isomorphe à $\mathcal{O}_{X}$. (Il suffit de remarquer que si $D \neq 0, D_{t} \neq 0$ car l'image de $D$ dans $S$ est ouvert, $D$ étant plat sur $S$, donc que $D_{t}$ est ample par le corollaire 1.)

Démonstration du ThÉoRÈme 2 . Soient $\mathscr{M}^{\prime}$ un $\mathcal{O}_{X^{\prime}}$-module inversible ample et $s$ le point fermé de $S$. Comme le sous-ensemble de $X^{\prime}$,

$$
Z=g\left(\cup_{j \in J} C_{j}\right) \cup \operatorname{Ass}\left(X_{s}^{\prime}\right)
$$

est fini, il existe un entier $m$ et une section $a \in \Gamma\left(X^{\prime}, \mathscr{M}^{\prime} \otimes m\right)$ tels que $Z \cong X^{\prime}{ }_{a}$ (EGA, II $\left.[2,4.5 .4]\right)$. Soient $\mathscr{M}=\mathscr{M}^{\prime} \otimes m$ et $D^{\prime}$ le diviseur correspondant à $\mathscr{M}$ et à $a$. Alors $D^{\prime} \cap Z=\varnothing$, donc $D^{\prime}$ est plat sur $S$, i.e. $D^{\prime} \in \operatorname{Div}^{+}\left(X^{\prime} \mid S\right)$.

Soit $U=X-\cup_{j \in J} C_{j}$. Comme $D^{\prime} \leqq g(U)$ et $g \mid U: U \rightarrow g(U)$ est un isomorphisme, $D=g^{*} D^{\prime} \in \operatorname{Div}^{+}(X / S)$. Soit $\mathscr{L}=\mathcal{O}_{X}(D)$. Il résulte du théorème 1 et de la construction de $D$ que

$$
r_{\mathscr{L}}: X \rightarrow P=\operatorname{Proj}\left(\oplus_{n \geqq 0} \Gamma(X, \mathscr{L} \otimes n)\right)
$$

est une contraction de $\left\{C_{j} ; j \in J\right\}$, et on en conclut par la proposition 4 .

Pour terminer, on va donner trois corollaires concernant les courbes sur un trait hensélien. Remarquons d'abord que toute courbe complète sur le spectre d'un anneau de valuation discrète hensélien est projective (EGA, IV [4, 21.9.13]), en particulier toute contraction d'une telle courbe est une contraction projective.

Corollatre 3. Soit $X$ une courbe complète sur un trait hensélien $S$. Alors tout morphisme de contraction $g: X \rightarrow X^{\prime}$ est égal (à un isomorphisme près) à $r_{O_{X}(D)}$ pour un $D \in \operatorname{Div}^{+}(X / S)$.

CoRollaIre 4. Soit $f: X \rightarrow S$ une courbe complète intègre sur un trait hensélien. Pour toute famille $\left\{C_{j} ; j \in J\right\}$ (distincte de l'ensemble de toutes les 
composantes irréductibles de la fibre fermée de f) il existe une contraction (projective) de $\left\{C_{j} ; j \in J\right\}$.

Démonstration. Soient $\left\{C_{i} ; i \in I\right\}$ les composantes irréductibles de la fibre fermée de $f$ et $J$ un sous-ensemble de $I$, distinct de $I$. On sait qu'il existe un diviseur effectif $\bar{D}$ sur la fibre fermée de $f$ tel que $\bar{D} \cap C_{j}=\varnothing$ pour tout $j \in J$ et tel que $\bar{D} \cap C_{i} \neq \varnothing$ pour $i \in I-J$ (EGA, IV [4, 21.9.7]).

Comme $S$ est hensélien, les conditions de (EGA, IV [4, 21.9.11]) sont satisfaites, donc il existe un diviseur $D$ sur $X$ tel que $D$ restreint à la fibre fermée soit égal à $\bar{D}$. On voit que $D \in \operatorname{Div}^{+}(X / S)$, donc $r_{\mathcal{O}_{X}(D)}$ donne une contraction projective de $\left\{C_{j} ; j \in J\right\}$, d'après le théorème 1 , comme $D \neq 0$ (Remarque 2).

Corollatre 5. Soit $f: X \rightarrow S$ une courbe intègre sur un trait hensélien. $I l$ existe une courbe projective $P$ sur $S$ et une $S$-immersion ouverte et schématiquement dense $X \rightarrow P$ telles que la fibre fermée de $X$ soit dense dans la fibre fermée de $P$.

Démonstration. D’après un résultat de Nagata [9] (généralisé, mais non publié par Mumford - cfr. [5, l'Appendice par Deligne, p. 413]) on sait que tout morphisme séparé et de type fini de schémas noethériens et intègres est " compactifiable ". Plus précisement, il existe une $S$-immersion ouverte et schématiquement dense $u: X \rightarrow X^{\prime}$ telle que $X^{\prime}$ soit un $S$-schéma propre.

Soient $s$ le point fermé de $S, t$ son point générique. Montrons que $X^{\prime}$ est une courbe sur $S$. Pour cela il suffit de montrer que $X^{\prime}$ est plat sur $S$, puisqu'on sait que $X^{\prime}{ }_{t}$ est irréductible et de dimension 1 .

$X^{\prime}$ est plat sur $S$ si et seulement si Ass $\left(X^{\prime}\right) \cong X^{\prime}{ }_{t}$ (voir [8, Lecture 6, Proposition 6]). Comme $X$ est plat sur $S$ et $X$ est schématiquement dense dans $X^{\prime}$,

$$
\operatorname{Ass}\left(X^{\prime}\right) \subseteq \operatorname{Ass}(X) \subseteq X_{t} \leqq X^{\prime}{ }_{t} .
$$

Soient $C=X^{\prime}{ }_{s}-X_{s}$ et $\left\{C_{j} ; j \in J\right\}$ les composantes irréductibles de dimension 1 de $C$. Le corollaire 4 montre qu'il existe une contraction projective $r: X^{\prime} \rightarrow P$ de $\left\{C_{j} ; j \in J\right\}$. Comme

$$
X \subseteq\left(X^{\prime}-U_{j \in J} C_{j}\right)
$$

et $r$ est un isomorphisme de $X^{\prime}-\bigcup_{j \in J} C_{j}$ sur $r\left(X^{\prime}-U_{j \in J} C_{j}\right)$, rou est une $S$-immersion ouverte et schématiquement dense. $X_{s}$ est dense dans $P_{s}$, parce que $X_{8}$ et $P_{8}$ sont des courbes algébriques et que $P_{8}-X_{8}$ est égal à un nombre fini de points fermés. 
AJoutí Pendant La CORReCtion Des ePReuves. On trouve aussi les résultats du théorème 1 et corollaire 4 dans J. Emsalem, Projectivité des schémas en courbes sur un anneau de valuation discrète, à paraitre dans Bull. Soc. Math. France.

\section{BIBLIOGRAPHIE}

1. A. Grothendieck et J. Dieudonné, Éléments de géométrie algébrique (EGA I), (Grundlehren Math. Wissensch. 166), Springer-Verlag, Berlin · Heidelberg · New York, 1971.

2. A. Grothendieck et J. Dieudonné, Éléments de géométrie algébrique (EGA II), Inst. Hautes Etudes Sci. Publ. Math. 8 (1961), 1-222.

3. A. Grothendieck et J. Dieudonné, Éléments de géométrie algébrique (EGA III), Inst. Hautes Etudes Sci. Publ. Math. 11 (1961), 1-167.

4. A. Grothendieck et J. Dieudonné, Éléments de géométrie algébrique (EGA IV), Inst. Hautes Etudes Sci. Publ. Math. 28 (1966), 1-255.

5. R. Hartshorne, Residues and duality, (Lecture Notes in Mathematics 20), SpringerVerlag, Berlin · Heidelberg · New York, 1966.

6. S. Kleiman, Toward a numerical theory of ampleness, Ann. of Math. 84 (1966), 293-344.

7. S. Lichtenbaum, Curves over discrete valuation rings, Amer. J. Math. 85 (1968), 380405.

8. D. Mumford, Lectures on curves on an algebraic surface, Annals of Math. Studies 59, Princeton University Press, Princeton, 1966.

9. M. Nagata, Imbedding of an abstract variety in a complete variety, J. Math. Kyoto Univ. 2-1 (1962), 1-10.

10. M. Raynaud, Faisceaux amples sur les schémas en groupes et les espaces homogènes (Lecture Notes in Mathematics 119), Springer-Verlag, Berlin · Heidelberg · New York, 1970.

11. O. Zariski, The theorem of Riemann-Roch for high multiples of an effective divisor on an algebraic surface, Ann. of Math. 76 (1962), 560-615. 